\title{
Postoperative Bare-Metal Stent Thrombosis 37 Days after Percutaneous Coronary Intervention: A Case Report
}

\author{
Ki Tae Jung MD ${ }^{\text {a, b }}$, Sang Hun Kim, MD, PhD*a, b , Keum Young So, MD, PhD ${ }^{\text {a, b }}$ \\ ${ }^{a}$ Department of Anesthesiology and Pain Medicine, Chosun University, School of Medicine, Dong-gu, \\ Gwangju, Republic of Korea \\ ${ }^{\mathrm{b}}$ Department of Anesthesiology and Pain Medicine, Chosun University Hospital, Dong-Gu, Republic \\ of Korea \\ *ksh3223@chosun.ac.kr
}

\begin{abstract}
Perioperative coronary stent thrombosis is a life-threatening complication for patients undergo noncardiac surgery early after stent implantation. We present a case of fatal stent thrombosis immediately after revision total knee replacement (TKR)in 71-year-old woman, who underwent multiple bare-metal stents (BMSs) implantation 37 days before surgery. She had previously undergone emergency surgery due to septic knee arthritis before BMS implantation. Dual-antiplatelet therapy with aspirin and clopidogrel were administered four week, and then aspirin alone was continued until the day of surgery. However, within 1 hour after revision $T K R$, total stent occlusion of the left anterior descending artery and near total thrombotic occlusion of obtuse marginal artery oswere developed due to the antiplatelet drug resistance, which is one of the strongly suspected predictor. Unfortunately she was died despite of the successful coronary blood flow recoveries.
\end{abstract}

Keywords: Antiplatelet, Coronary artery, Drug resistance, Noncardiac surgery, Stent thrombosis

\section{INTRODUCTION}

The anesthesiologists are frequently faced with patients who need an elective or semi-urgent surgery and are on dual-antiplatelet therapy (DAPT) due to a recent coronary artery stent implantation. They also should determine the date of the surgery and whether DAPT should be continued, modified, or discontinued with the cardiologist and surgeon. Generally, Patients, who need elective noncardiac surgery in the subsequent 12 months, probably indicate a strategy of balloon angioplasty or bare-metal stent (BMS) implantation followed by 4 to 6 weeks of DAPT[1, 2]. On development of stent thrombosis, there were several predictors such as left main or multivessel stenting, poor ventricular function $(<30 \%)$, chronic renal failure, diabetes, cancer, surgery, antiplatelet therapy discontinuation, and antiplatelet therapy resistance [2-4]. We present a case of fatal multiple BMS thrombosis immediately after revision total knee replacement (TKR), in patient who underwent BMS implantation 37 days before revision TKR and had several strong predictors mentioned above. The preparation and submission of this case report was approved by the Chosun University Hospital Institutional Review Board (CHOSUN 2015-01-008).

\section{CASE REPORT}

A 71-year-old woman underwent the revision TKR 37 days after BMSs implantation at the proximal to the mid parts of left anterior descending artery (p-to-m LAD), obtuse marginal artery (OM) of left circumflex artery (LCX), and the proximal part of right coronary artery (pRCA). She had hypertension and diabetes for 10 years. She underwent emergent removal surgery of septic knee prostheses 5 day before BMSs implantation. At that time, preoperative erythrocyte sedimentation rate [ESR: $69 \mathrm{~mm} / \mathrm{hr}(\mathrm{NR}:<30)$ ] and C-reactive protein [CRP: $1.25 \mathrm{mg} / \mathrm{dL}(\mathrm{NR}:<0.3)]$ was increased with normal prothrombin time [PT: $12.1 \mathrm{sec}$ (NR: 9.4-12.5)], activated partial thromboplastin time [aPTT: $31.6 \mathrm{sec}$ (NR: 28-44)], and platelet count [234,000/ $\mu$ l (NR: 150,000-400,000)]. Preoperative electrocardiogram (ECG) and laboratory findings were suggestive of acute myocardial infarction without clinical findings such as chest pain and dyspnea. Postoperative transthoracic echocardiogram (TTE) showed old myocardial infarction with an ejection fraction of 38.Her plasma level of serum troponin $\mathrm{T}$, creatine kinase $\mathrm{MB}$ isoenzyme, and $\mathrm{N}$-terminal pro-brain natriuretic peptide were 0.116 
$\mathrm{ng} / \mathrm{mL}$ [normal range $(\mathrm{NR}):<0.013 \mathrm{ng} / \mathrm{ml}], 3.979 \mathrm{ng} / \mathrm{mL}(\mathrm{NR}:<3.77 \mathrm{ng} / \mathrm{ml})$, and $6971 \mathrm{pg} / \mathrm{mL}(\mathrm{NR}:<$ $262 \mathrm{ng} / \mathrm{ml}$ ), respectively. Coronary angiography (CAG), 5 postoperative days, showed diffuse $80-100 \%$ stenosis at the several parts of LAD, diffuse $90 \%$ stenosis at OM of LCX, and diffuse $90 \%$ stenosis at pRCA with an ejection fraction of $30 \%$ (figure $1 \mathrm{a}, \mathrm{b}$ ). In view of the angiographic findings, BMSs were successfully deployed at the stenotic regions of coronary arteries (figure $1 \mathrm{c}, \mathrm{d}$ ). Dualantiplatelet therapy with Aspirin 100mg/d and clopidogrel $75 \mathrm{mg} / \mathrm{d}$ were administered four week, and then aspirin alone was continued until the day of surgery. One week before revision TKR, the increased ESR $(38 \mathrm{~mm} / \mathrm{hr})$ and CRP $(0.66 \mathrm{mg} / \mathrm{dL})$ were sustained with normal WBC and neutrophil count despite she was treated with vancomycin to control infection. PT, aPTT, and platelet count were $15.2 \mathrm{sec}, 24.8 \mathrm{sec}$, and 195000/ $\mu$ l, respectively. One day before revision TKR, platelet function was evaluated by the PFA-100 ${ }^{\circledR}$ (Platelet Function Analyzer, Dade Behring, Leiderbach, Germany); closure time with a collagen/EPI cartridge and a collagen/ADP cartridge was $186 \mathrm{sec}$ (NR: 94-193) and $143 \mathrm{sec}$ (NR: 71-118). In the operating room, general anesthesia was induced with propofol and rocuronium followed by maintenance with sevoflurane, remifentanil, and a 50\% oxygen-air mixture uneventfully. 30 minutes after end of the surgery,sudden chest pain and dyspnea were developed followed with severe hypotension $(40 /-\mathrm{mmHg}$ ), which was not controlled with intermittent bolus atropine and phenylephrine intravenous injection as well as continuous dopamine and norepinephrineinfusion. Serum troponin $\mathrm{T}$, blood pressure, heart rate, and peripheral oxygen saturation were $6.940 \mathrm{ng} / \mathrm{mL}, 60 / 50 \mathrm{mmHg}, 90$ beats/min, and 89\%, respectively. Urgent CAG1 hour after events showed total stent occlusion of pLAD by thrombosis and near total thrombotic occlusion of OM os ofLCX(figure2 a, b).Unfortunately, she was died due to persistent cardiogenic and hypovolemic shock, even though the blood flows of LAD and LCX were successfully recovered by kissing balloon inflation and clopidogrel $600 \mathrm{mg}$ was restarted during CAG (figure2 c).
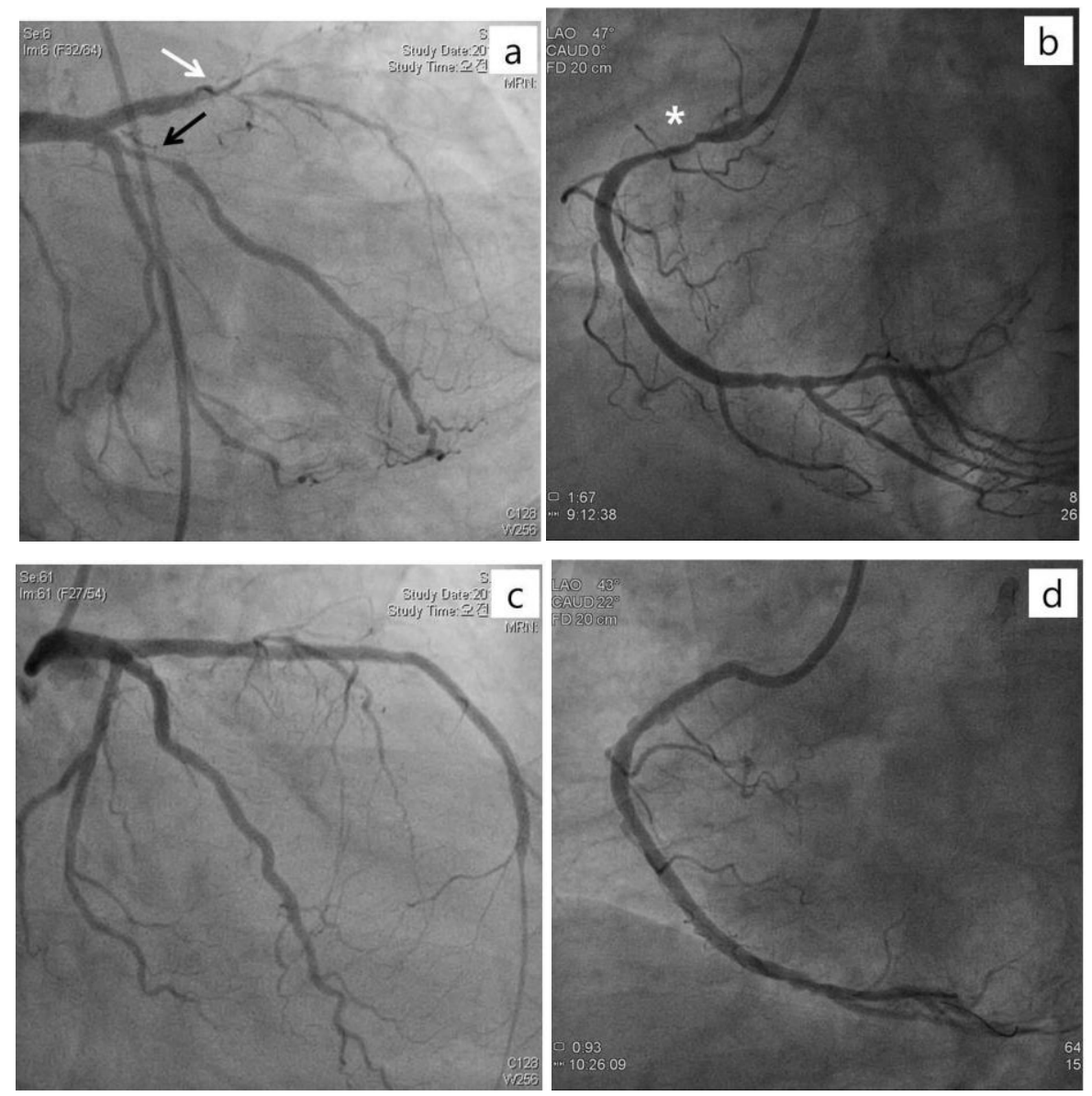

Figure1. Elective Coronary Angiography before Noncardiac Surgery. Top: Right Anterior Oblique Caudal View of the Proximal to Mid Parts of Left Anterior Descending Arterystenosis (a, White Arrow) and Obtuse Marginal Artery of Left Circumflex Artery Stenosis (a, Black Arrow), and Left Anterior Oblique View of the Right Coronary Artery Stenosis (b, White Asterisk). Bottom: Angiographic Results That Their Coronaries Were Successfully Reperfused After Percutaneous Coronary Intervention With Implantations of Bare-Metal Stents (c, d). 

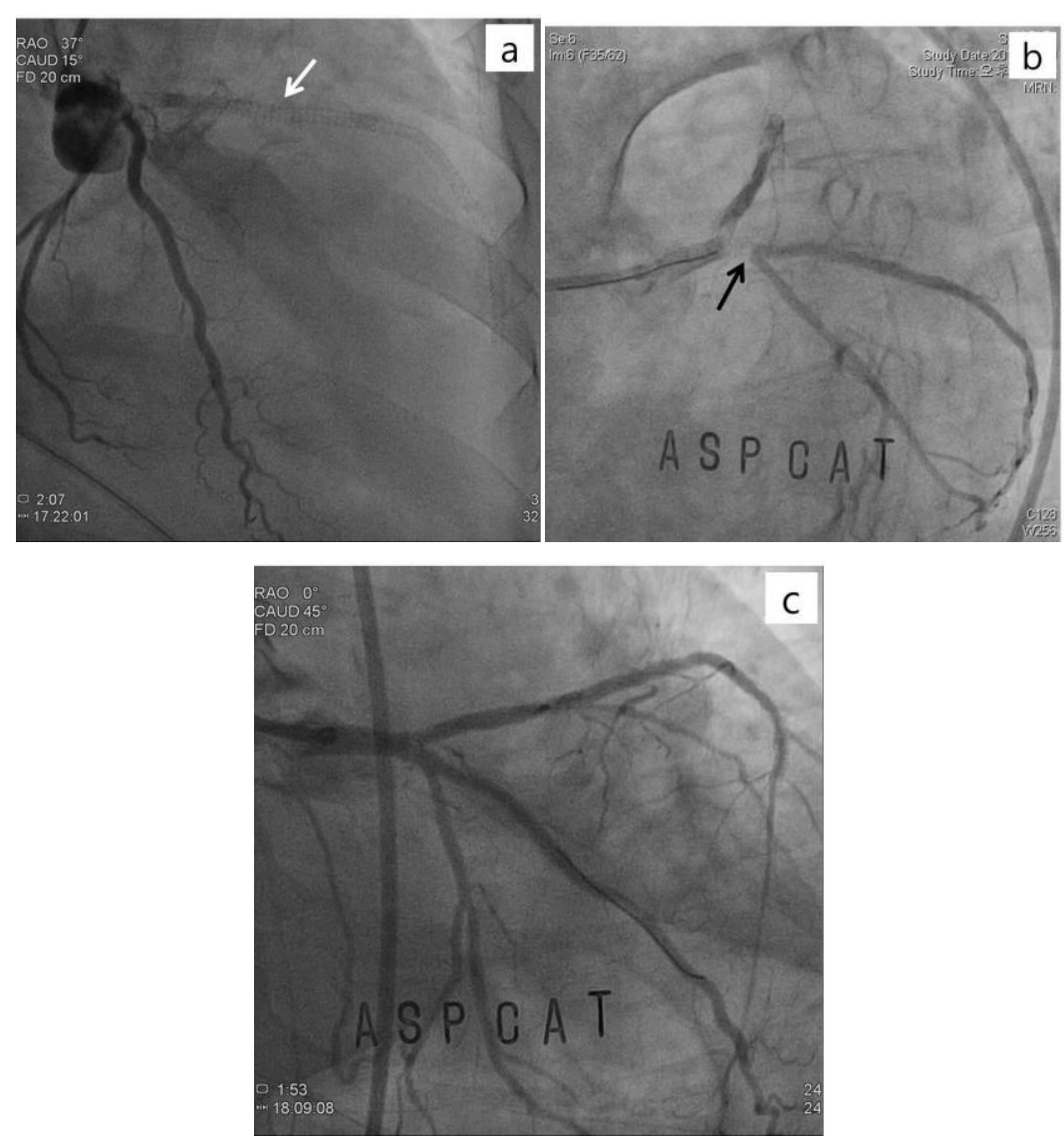

Figure2. Urgent Coronary Angiography after Noncardiac Surgery. It Showed Total Stent Occlusion of the Proximal Parts of Left Anterior Descending Artery by Thrombosis in Right Anterior Oblique Caudal View (a, White Arrow) and Near Total Thrombotic Occlusion of Obtuse Marginal Artery Os of Left Circumflex Artery in Left Anterior Oblique Cranial View (b, Black Arrow). The Blood Flows of LAD And LCX Were Successfully Recovered by Kissing Balloon Inflation (c).

\section{DisCUSSION}

Anesthesiologists commonly are in a dilemma how long the elective surgery should be postponed and whether DAPT should be continued, modified, or discontinued before noncardiac surgery (NCS) to avoid perioperative bleeding orstent thrombosis. On development of stent thrombosis, there were several predictors such as left main or multivessel stenting, poor ventricular function $(<30 \%)$, chronic renal failure, diabetes, cancer, surgery, antiplatelet therapy discontinuation, and antiplatelet therapy resistance[2-4].Our patient also had several predictors of them, and we suspected that some predictors might strongly contribute in stent thrombosis formation.

First, antiplatelet therapy resistance seems to be the strongest suspected predictor among them in this patient. Lemesles et al. [4] documented that patients presenting with stent thrombosis had single or combined resistance of aspirin and clopidogrel, of which the rate reported 5.5-43\% and $11-44 \%$, respectively [4]. They also suggested that patients with low response to antiplatelet therapy, and especially a low response to clopidogrel, were at higher risk of early cardiovascular events. While, Wenaweser et al. [5] showed that resistance of aspirin and the combination of aspirin and clopidogrel may play an important role in the formation of stent thrombosis using optical aggregometry, and suggested that aspirin but not clopidogrel resistance was mainly associated with stent thrombosis. Our patient also showed aspirin resistance, which can be supported by normal closure time with a collagen/EPI cartridge even though we followed the recommended duration and doses of aspirin and clopidogrel. Interestingly, results of platelet function test by the PFA- $100^{\circledR}$ also showed that clopidogrel did not overcome aspirin resistance like results of Wenaweser's study [5].

Second, we are well-known that inappropriate antiplatelet therapy discontinuation is the strongest suspected predictor among them [4]. Therefore, most authors recommended that thienopyridines (ticlopidine or clopidogrel) with aspirin should be administered for 4 weeks after implantation of BMSs followed with daily aspirin therapy unless contraindicated, and then the NCS can be performed 
1 week later after discontinuation of thienopyridine therapy[2, 6, 7]. However, this recommended duration of antiplatelet therapy or NCS postponement is somewhat arbitrary because they could not provide the strong evidence. Some authors suggested that it is better to postpone the NCS as long as possible and to continue DAPT more than the recommended duration after BMS implantation [8-10]. They documented that the earliest optimal time for intermediate-to-high-risk elective NCS was 46 to 180 days [8], and the NCS within 6 weeks, especially in patients revascularized after an acute coronary syndrome, led to the more frequent perioperative death and myocardial ischemic events [9]. Similar with our case report, Sekiya et al. [10] reported that $100 \%$ thrombotic stenosis of BMS was developed soon after total gastrectomy with discontinuation of DAPT 37 days after stent implantation.

Finally, there are some minor suspected predictors such as multi vessel stent, diabetes, previous surgery, and septic knee arthritis. Especially, it is well known that sepsis-induced hypercoagul ability can be contributed to the development of stent thrombosis by procoagulant upregulation and anticoagulant impairment [11]. However, our patient was not diagnosed with generalized sepsis or systemic inflammatory response syndrome except septic knee arthritis, which was well controlled with intravenous vancomycin therapy even though the increased ESR and CRP were sustained. Surgery itself also can increase the platelet activity, by which postoperative hypercoagul ability can be induced and occur for up to a week afterwards[3, 4, 12].Generally, the platelet count and spontaneous fibrinolytic activity are decreased in the first days postoperatively, and then subsequently the platelet count as well as the plasminogen concentration increase and spontaneous fibrinolytic activity gradually return to normal in the postoperative period [13]. Our patients also showed almost a twofold increased trend of platelet count [478000/ $\mu 1$ (NR: $150000-400000 / \mu 1)]$ within postoperative 10 days followed with return to normal before revision TKR. So, we thought that these minor predictors did not directly contribute in stent thrombosis formation.

\section{Conclusion}

In conclusion, our case report showed that the recommended duration (4 to 6 weeks) of DAPT for elective NCS is the risky window period, which is not sufficient to prevent the stent thrombosis in patients with several strong predictors mentioned above. Therefore, we cautiously suggest that it may be better to defer NCS as long as possible until the predictors are fully evaluated and controlled, especially, platelet function test should be performed during DAPT for screening and management of the antiplatelet drug resistances.

\section{REFERENCES}

[1] Levine GN, Bates ER, Blankenship JC, Bailey SR, Bittl JA, Cercek B et al. 2011 ACCF/AHA/SCAI Guideline for Percutaneous Coronary Intervention: a report of the American College of Cardiology Foundation/American Heart Association Task Force on Practice Guidelines and the Society for Cardiovascular Angiography and Interventions. Circulation 2011; 124: e574-651.

[2] Fleisher LA, Beckman JA, Brown KA, Calkins H, Chaikof E, Fleischmann KE et al. ACC/AHA 2007 Guidelines on Perioperative Cardiovascular Evaluation and Care for Noncardiac Surgery: Executive Summary: A Report of the American College of Cardiology/American Heart Association Task Force on Practice Guidelines (Writing Committee to Revise the 2002 Guidelines on Perioperative Cardiovascular Evaluation for Noncardiac Surgery): Developed in Collaboration With the American Society of Echocardiography, American Society of Nuclear Cardiology, Heart Rhythm Society, Society of Cardiovascular Anesthesiologists, Society for Cardiovascular Angiography and Interventions, Society for Vascular Medicine and Biology, and Society for Vascular Surgery. Circulation 2007; 116: 1971-96.

[3] Jones C, Liban B. Anaesthesia and coronary artery stents. Curr Anaesth Crit Care 2009; 20: 1504.

[4] Lemesle G, Delhaye C, Bonello L, de Labriolle A, Waksman R, Pichard A. Stent thrombosis in 2008: Definition, predictors, prognosis and treatment. Arch Cardiovasc Dis 2008; 101: 769-77.

[5] Wenaweser P, Dörffler-Melly J, Imboden K, Windecker S, Togni M, Meier B et al. Stent thrombosis is associated with an impaired response to antiplatelet therapy. J Am Coll Cardiol 2005; 45: 1748-52.

[6] Wilson SH, Rihal CS, Bell MR, Velianou JL, Holmes DR, Jr., Berger PB. Timing of coronary stent thrombosis in patients treated with ticlopidine and aspirin. Am J Cardiol 1999; 83: 1006-11. 
[7] Berger PB, Bell MR, Hasdai D, Grill DE, Melby S, Holmes DR, Jr. Safety and efficacy of ticlopidine for only 2 weeks after successful intracoronary stent placement. Circulation 1999; 99: 248-53.

[8] Wijeysundera DN, Wijeysundera HC, Yun L, Wasowicz M, Beattie WS, Velianou JL et al. Risk of elective major noncardiac surgery after coronary stent insertion: a population-based study. Circulation 2012; 126: 1355-62.

[9] Cruden NL, Harding SA, Flapan AD, Graham C, Wild SH, Slack R et al. Previous coronary stent implantation and cardiac events in patients undergoing noncardiac surgery. Circ Cardiovasc Interv 2010; 3: 236-42.

[10] Sekiya Y, Takahashi H, Tanaka M. Patient who developed acute myocardial infarction after totalgastrectomy. Masui 2010; 59: 210-2.

[11] Simmons J, Pittet JF. The coagulopathy of acute sepsis. Curr Opin Anaesthesiol 2015; 28: 227-36.

[12] Mahla E, Lang T, Vicenzi MN, Werkgartner G, Maier R, Probst C et al. Thromboelastography for monitoring prolonged hypercoagulability after major abdominal surgery. Anesth Analg 2001; 92: 572-7.

[13] Ygge J. Changes in blood coagulation and fibrinolysis during the postoperative period. Am J Surg 1970; 119: 225-32? 